\title{
Silent night
}

Your partner might have suggested all kinds of things, from nasal strips to eating honey before going to bed, but did it stop you snoring? In the Journal of Biomechanics (doi:10.1016/j.jbiomech.2006.03.022; 2006), Z. S. Liu and colleagues offer fresh, quantitative insight into 'obstructive sleep apnea' that could lead to a quieter night.

Liu et al. have developed a three-dimensional finite-element model of the human head to analyse tissue vibrations in response to pressure loads. They confirm that the soft palate - already a prime suspect - is the strongest sound source throughout the relevant frequency range of $10-60 \mathrm{~Hz}$. The tongue only contributes significantly at about $20 \mathrm{~Hz}$, but the hard palate, on the other hand, is found to act as a 'snoring sink'.

Although their model is, at this point, rather rough, Liu et al. hope that it can be developed further using anatomical data from snorers. Understanding the mechanics could mean that it is possible to avoid excitation of the trouble-making regions in the head — and that longsuffering partners might at last get a good night's sleep.

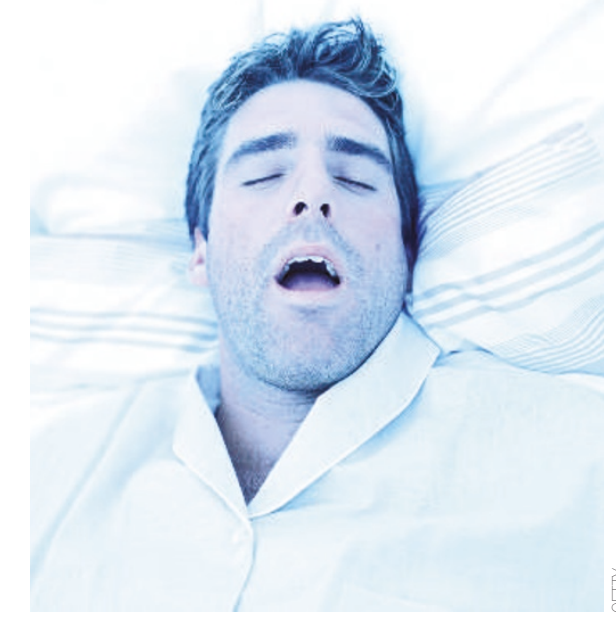

\section{Non-magnetic spin polarization}

The fundamental unit of conductance $\left(2 e^{2} / h\right)$ through a $1 \mathrm{D}$ ballistic conductor, such as a nanowire or nanotube, is made up of equal contributions from spin-up and spin-down electrons. Separating these two components usually requires a magnetic field - without it, the energy of the two spin states is identical.
But, according to Rolf Crook and colleagues, this needn't always be the case (Science 213, 1359-1362; 2006).

For a 1D channel electrostatically patterned into a GaAsbased 2D electron system, Crook et al. observe a plateau in the zero-magneticfield conductance characteristics, at exactly half the fundamental unit of conductance. This suggests that the electrons within the channel become spontaneously spinpolarized. This ability to generate a spinpolarized current, without the use of a magnetic field to lift the usual spin degeneracy, could prove valuable in the emerging field of spintronics.

\section{GRAVITATIONAL GET-TOGETHER}

The hunt is on for gravitational waves, thought to be generated as neutron stars in a binary system spiral in towards each other and coalesce. Although the latest result from two of the terrestrial interferometers, LIGO and TAMA300, is another limit on the rate of inspiral events, the combined analysis of data from these experiments sets a new precedent (B. Abbott et al. Phys. Rev. D 73, 102002; 2006).

TAMA300 is a $300-\mathrm{m}$ interferometer in Tokyo, Japan; the US-based LIGO network consists of a 4-km detector in Louisiana, and 4-km and 2-km detectors in Washington state. Looking for coincident signals in the data from the two facilities reduces the rate of false alarms; it also improves the sky coverage and the chances of localizing any source of gravitational waves. Furthermore, the observation time is lengthened, as only one of the LIGO interferometers need be in operation.

LIGO and TAMA300 present an upper limit ( $90 \%$ confidence level) on the number of neutron-star inspirals at 49 per year 'per Milky-Way-equivalent galaxy' — comparable to the limit set by LIGO alone on a separate data set. Coincidence searches are now planned with two other interferometers, GEO 600 in Germany and Virgo in Italy.

\section{A repulsive couple}

We expect attractive forces to bind particles together and repulsive ones to separate them, but Klaus Winkler and colleagues show that this intuition can be misleading (Nature 441, 853-856; 2006). They trap rubidium atoms in a three-dimensional optical lattice, and find that pairs of atoms can form stable composites - even though in free space the two atoms would have repelled each other.

The key to understanding this behaviour is that the particles, once brought together at one site of the optical lattice, are unable to convert their potential energy (which is higher than the energy of the separated atoms) into kinetic energy - a process that would lead to a decay of the pair. Instead, the couple is forced to stay together, even when changing position in the lattice.

In a conventional solid, lattice vibrations result in rapid dissipation of the excess energy of a repulsive pair, making the alliance unstable. Winkler et al. expect that their experimental realization of repulsively bound atom pairs could be the starting point to explore other exotic many-body states and quantum phases.

\section{Random walk to the fridge}

Thermal fluctuations are usually a nuisance when it comes to measuring small objects at low temperature. However, Christian van den Broeck and Ryoichi Kawai have now found a purpose for them. They propose to use thermal fluctuations as a cooling mechanism (Phys. Rev. Lett. 96, 210601; 2006).

The authors' refrigeration concept is based on a brownian motor composed of a triangular wedge (similar to an arrowhead) in one reservoir, connected rigidly to a flat paddle in a lower reservoir. The wedge and paddle move horizontally as one unit. Particles strike the paddle randomly, but in the upper reservoir, the momentum is dissipated aymmetrically by the wedge. A net momentum produces motion in the direction of the arrow-head.

If an external force moves the motor against this direction, the system will react with an opposing force (Le Chatelier-Braun principle). In other words, the system

will raise the temperature of the lower reservoir by inducing a heat flow from the upper reservoir, thus cooling it. The authors calculate a cooling of $1^{\circ}$ per minute for an aqueous reservoir of diameter $0.1 \mu \mathrm{m}$ — sufficient for molecules within lipid bilayers.

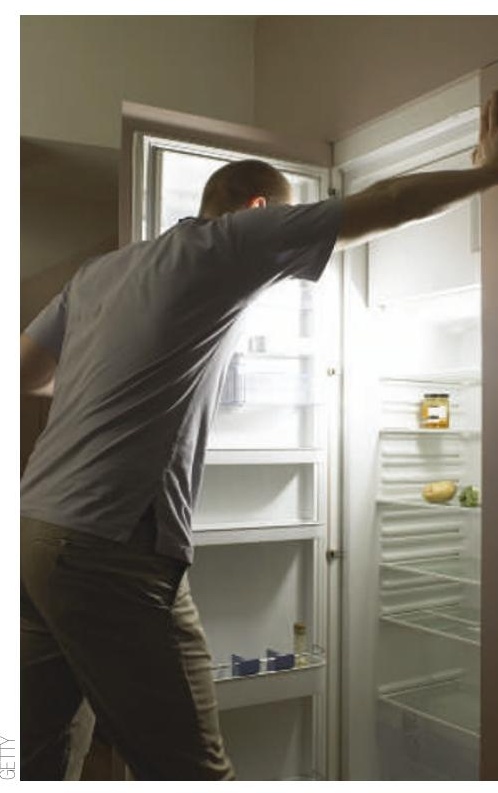

